\title{
Fiber Optic Coupled Raman Based Detection of Hazardous Liquids Concealed in Commercial Products
}

\author{
Michael L. Ramírez-Cedeño, ${ }^{1,2}$ Natalie Gaensbauer, ${ }^{3}$ Hilsamar Félix-Rivera, ${ }^{1}$ \\ William Ortiz-Rivera, ${ }^{1}$ Leonardo Pacheco-Londoño, ${ }^{1}$ and Samuel P. Hernández-Rivera ${ }^{1}$ \\ ${ }^{1}$ ALERT-DHS Center of Excellence, Center for Chemical Sensors Development, Department of Chemistry, \\ University of Puerto Rico-Mayagüez, P.O. Box 9000, Mayagüez, PR 00681-9000, USA \\ ${ }^{2}$ U.S. Department of the Treasury, Bureau of Engraving and Printing, Washington, DC 20228, Boston, USA \\ ${ }^{3}$ Mechanical and Industrial Engineering Department, Northeastern University, 360 Huntington Avenue, Boston, \\ MA 02115-5000, USA
}

Correspondence should be addressed to Samuel P. Hernández-Rivera, samuel.hernandez3@upr.edu

Received 15 July 2011; Revised 1 October 2011; Accepted 7 October 2011

Academic Editor: Maher S. Amer

Copyright (C) 2012 Michael L. Ramírez-Cedeño et al. This is an open access article distributed under the Creative Commons Attribution License, which permits unrestricted use, distribution, and reproduction in any medium, provided the original work is properly cited.

\begin{abstract}
Raman spectroscopy has been widely proposed as a technique to nondestructively and noninvasively interrogate the contents of glass and plastic bottles. In this work, Raman spectroscopy is used in a concealed threat scenario where hazardous liquids have been intentionally mixed with common consumer products to mask its appearance or spectra. The hazardous liquids under consideration included the chemical warfare agent (CWA) simulant triethyl phosphate (TEP), hydrogen peroxide, and acetone as representative of toxic industrial compounds (TICs). Fiber optic coupled Raman spectroscopy (FOCRS) and partial least squares (PLS) algorithm analysis were used to quantify hydrogen peroxide in whiskey, acetone in perfume, and TEP in colored beverages. Spectral data was used to evaluate if the hazardous liquids can be successfully concealed in consumer products. Results demonstrated that FOC-RS systems were able to discriminate between nonhazardous consumer products and mixtures with hazardous materials at concentrations lower than $5 \%$.
\end{abstract}

\section{Introduction}

In August 2006, a terrorist plot to destroy aircrafts on transatlantic flights was discovered and timely stopped in London. The plan involved the use of liquid explosives stored in beverage bottles that would pass checkpoints without being detected [1]. The liquids were going to be mixed in flight to create an improvised explosive device (IED) that was going to be left in the aircraft and detonated remotely after the aircraft landed. Immediately after the event, airport security agencies in the United Kingdom and the United States of America established a ban on all liquids except medicines and infant food beyond checkpoints. The position of airport security officials has changed several times to allow certain amount of liquid or gel-based products. However, when or how terrorists would try to pass hazardous liquids into ports, government buildings, or public areas to generate threats using explosives or chemical agents is unpredictable.
The possibility of using liquid explosives for terrorism purposes is accompanied by the use of chemical warfare agents (CWAs) or toxic industrial compounds (TICs) for the same purposes. Such chemicals, which can be accessed in large quantities, can be extremely toxic on contact or inhalation even at small quantities $[2,3]$. The establishment of current regulations also recognizes the challenges of detecting dangerous liquids when hidden or mixed within common nondangerous products. For this reason, it is important to develop methodologies that would enable to differentiate between common products and compounds that can be combined for terrorism intents [4].

Normal or spontaneous Raman spectroscopy (RS) is one of the most promising tools under consideration [5-7]. Current systems are portable, sensitive, and have a wide variety of accessories to tailor applications in the field and in the laboratory [8-10]. The technique is based on inelastic scattering which brings information on vibrational signatures 
of molecules; therefore, it can be used as a specific means of detection. One of the strengths of RS is that it is able to analyze samples through various transparent glass and plastic containers [11-14]. This allows noninvasive, nondestructive inspection of the content of a container without opening minimizes exposure to potentially harmful substances and helps to speed the screening process. Since water does not present a strong signature in RS measurements, such systems are able to characterize aqueous-based commercial products. In addition, RS data can be successfully combined with chemometrics algorithms such as principal components analysis (PCA), partial least squares (PLS) regression analysis, and discriminant analysis (DA) for improved detection and quantification in a variety of matrices [15-17].

Principal components analysis (PCA) is very useful as a data reduction technique for spectroscopic analysis. In PCA, spectra are projected in space as orthogonal variables known as vectors or principal components (PCs). The goal is to capture as much variance as possible with the least amount of vectors (PCs) while reducing unwanted noise by transferring to less contributing PCs. Usually data is pretreated to smooth random differences and to bring out subtle differences not perceivable by simple evaluation of spectra by eye sight. Each component has a loading and a score. The loading describes the spectral features in the original spectra that are captured by the component, and the score tells how the spectrum of each PC compares/contributes to the original (experimental) spectra in terms of that particular component by assigning a value. Data is then visualized as a plot of scores which shows how different or similar are samples, data groups (classes), or the whole data set. In PLS, the variation observed during a PCA regression analysis is correlated to changes in concentration or other measurable property performing a regression of data variance $(x)$ versus a known variable $(y)[18,19]$.

In this study, FOCRS was used to characterize samples in which hazardous liquids were concealed in commercial products by mixing them with common liquids in their original containers. Common commercial products such as liquors, perfumes, and beverages can exhibit color or chemical composition that can mask the presence of the contaminant either by spectral congestion or more critically by exhibiting endogenous fluorescence. Hydrogen peroxide was used as a simulant for peroxide-based materials or precursors that can be used as homemade explosives. TEP is a structural analog of a CWA and therefore is commonly used as a chemical simulant for it. Acetone was used as representative of industrial solvents classified as TICs, but it is also a precursor of the family of cyclic organic peroxides collectively referred to as acetone peroxide homemade explosives. The results suggested that FOCRS can be used to discriminate and quantify the hazardous liquid concealed in the commercial products.

\section{Experimental Section}

2.1. Materials and Reagents. The commercial liquid products employed in this study were Gatorade Frost Glacier Freeze (blue color), Fruit Punch (red color) and Orange (orange color), Dewar's White Label whiskey, Ron Bacardi light

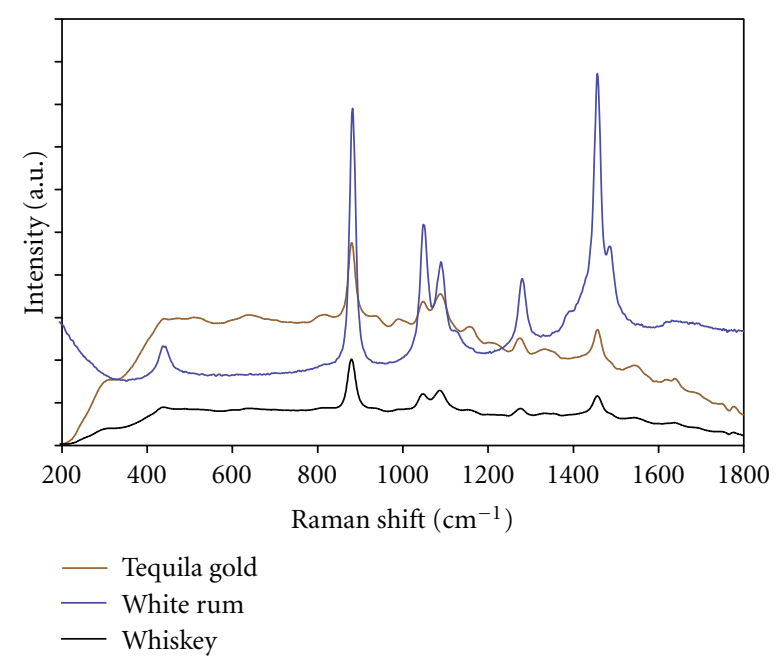

FIGURE 1: Raman spectrum of tequila, rum, and whiskey acquired with FOCRS. Parameters were $785 \mathrm{~nm}$ laser, $200 \mathrm{~mW}$, and $2 \mathrm{~s}$ integration time. All liquors exhibited similar spectra preserving spectral features.

rum, Tequila Cuervo Gold, and Ralph perfume. Hydrogen peroxide (50 wt. $\%$ in water) and acetone (99+\%) were obtained from Fisher Scientific International, Chicago, IL, USA. Cyclohexane ( $\geq 99 \%$ ) and triethyl phosphate (TEP; $\geq 99 \%$ ) were obtained from Sigma-Aldrich Chemical Company (St. Louis, MO, USA).

2.2. Measurements and Analysis. Portable FOC-based instrument (Raman Systems model R-3000 HR) was used to evaluate the content of commercial products. The RS system had an excitation wavelength of $785 \mathrm{~nm}$ (red) with $250 \mathrm{~mW}$ maximum output power measured at the head of the probe. The spectra of the original liquid in its container were collected though the container walls. The liquid was then replaced with $30 \mathrm{~mL}$ of a hazardous liquid and analyzed. Liquids were mixed at random volume to volume ratios $(\mathrm{v} / \mathrm{v})$ to prepare a total of $50 \mathrm{~mL}$. Raman spectra were acquired from $200 \mathrm{~cm}^{-1}$ to $1800 \mathrm{~cm}^{-1}$. The system was calibrated using HPLC grade cyclohexane as an external standard by measuring the band at Raman shift location about $801.3 \mathrm{~cm}^{-1}$. The data was exported and manipulated using Grams v.9 (Thermo Fisher Scientific, Waltham, MA, USA). Pretreatments and PLS analyses and other calculations were performed using MATLAB v.8 (The Mathworks, Natick, MA, USA.), and PLS Toolbox Solo v. 6.3 (Eigenvector Technologies, Wenatchee, WA, USA.), and Excel v. 2010 (Microsoft, Redmond, WA, USA).

\section{Results and Discussion}

3.1. Detection of Peroxide in Liquors. The spectra of the three liquors: whiskey, rum, and tequila were obtained in their original clear glass bottle. The results are presented in Figure 1. The spectra of the liquors were substantially similar although there were some differences in relative peak intensities. The additives that render the yellowish (gold) 


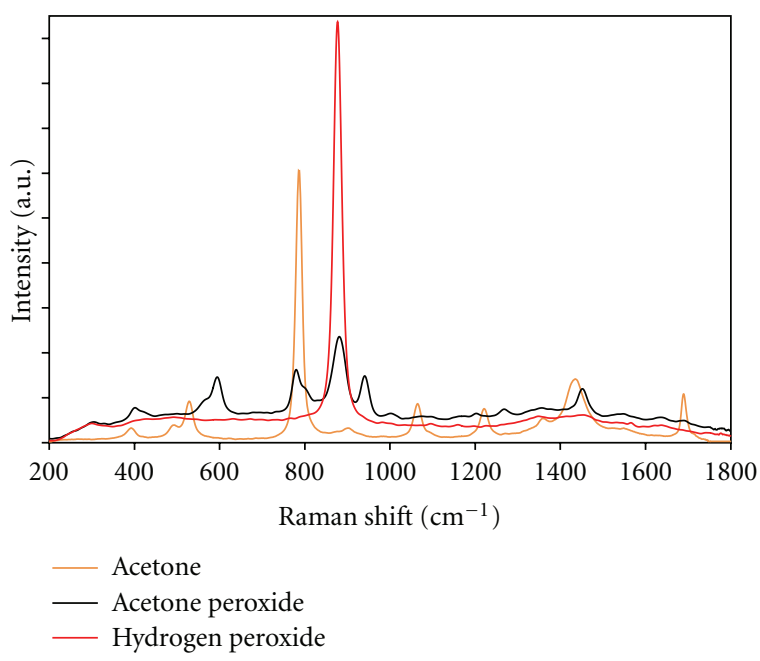

Figure 2: Raman spectra of acetone, hydrogen peroxide, and an acetone peroxide mixture contained in a clear glass bottle. Parameters were $785 \mathrm{~nm}, 10 \mathrm{~s}$ acquisition time at $200 \mathrm{~mW}$ of laser power. The characteristic peak for acetone is located at $798 \mathrm{~cm}^{-1}$ and for peroxide at $878 \mathrm{~cm}^{-1}$. The main acetone peroxide peaks are located at $588 \mathrm{~cm}^{-1}, 780 \mathrm{~cm}^{-1}, 890 \mathrm{~cm}^{-1}$, and $936 \mathrm{~cm}^{-1}$.

color of the tequila and whiskey presented some fluorescence identified by the shift in the baseline of the spectra. However, it was possible to identify the main peaks associated with the alcohol content. These spectra as well as the spectrum for ethanol, the main component of hard liquors, present peaks at $881 \mathrm{~cm}^{-1}$ (C-C-O symmetric stretch), $1048 \mathrm{~cm}^{-1}$ (C-C-O asymmetric stretch) and $1086 \mathrm{~cm}^{-1}$ (C-O stretch), $1280 \mathrm{~cm}^{-1}\left(\mathrm{C}-\mathrm{CH}_{3}\right.$ deformation), and $1456 \mathrm{~cm}^{-1}$ (C-H stretch in $\mathrm{CH}_{2}$ and $\left.\mathrm{CH}_{3}\right)$ [20]. Then the contents of the clear glass bottle were replaced with acetone and hydrogen peroxide so that the contents appeared to be clear rum. These consumer products are easily acquired at beauty supplies and drugstores, but they are also precursors of acetone-peroxide, a well-known homemade explosive (HME) [21,22].

The Raman spectra of acetone, peroxide, and acetoneperoxide are presented in Figure 2. The spectrum of acetone showed high-intensity peaks at $530 \mathrm{~cm}^{-1}(\mathrm{C}=\mathrm{O}$ deformation), $789 \mathrm{~cm}^{-1}$ (C-C symmetric stretch), $1225 \mathrm{~cm}^{-1}$ (C-C asymmetric stretch), and $1691 \mathrm{~cm}^{-1}(\mathrm{C}=\mathrm{O}$ stretch band). The most prominent vibrational Raman signature is located at $789 \mathrm{~cm}^{-1}$ [23]. Hydrogen peroxide is characterized by a strong peak at $878 \mathrm{~cm}^{-1}$, characteristic of the $\mathrm{O}-\mathrm{O}$ stretching mode. The main acetone peroxide peaks are located at $400 \mathrm{~cm}^{-1}$ (ring breathing), $594 \mathrm{~cm}^{-1}$ (O-C-O bending), $779 \mathrm{~cm}^{-1}$ (O-C-O symmetric stretch and Me-C-Me symmetric stretch), $880 \mathrm{~cm}^{-1}$ (O-O stretch and Me rocking synchronous and asynchronous), $940 \mathrm{~cm}^{-1}$ (C-C stretch), $1067 \mathrm{~cm}^{-1}$ (C-C and ring $\mathrm{C}-\mathrm{O}$ stretch), and $1451 \mathrm{~cm}^{-1}(\mathrm{H}-$ $\mathrm{C}-\mathrm{H}$ bend) [22-24]. The position, intensity, and uniqueness of the major peaks in acetone and peroxide make them easily detected by RS and can be used as markers for this explosive mixture $[24,25]$.

In Figure 3, the acetone and peroxide spectroscopic signatures are compared to those of rum. The rum spectrum

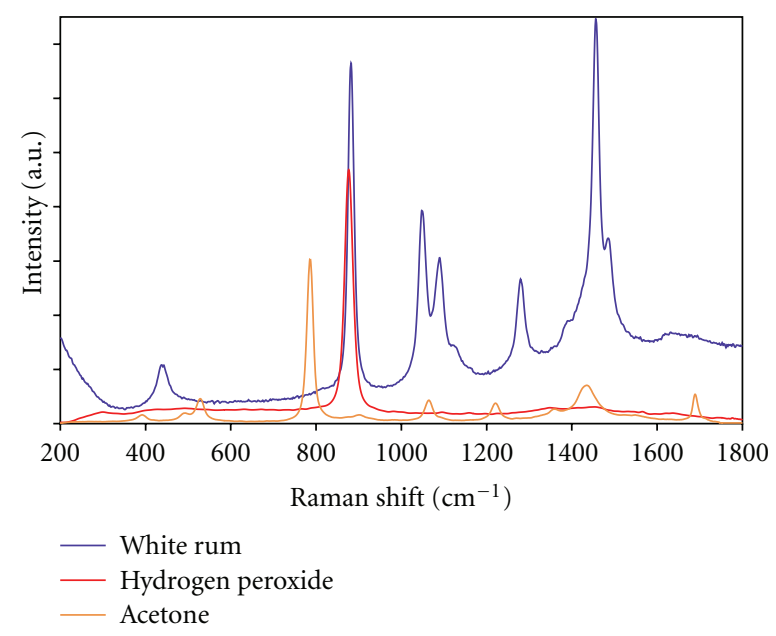

Figure 3: Spectroscopic signatures of rum versus acetone and peroxide. Parameters were $785 \mathrm{~nm}, 10 \mathrm{~s}$, and a laser power of $200 \mathrm{~mW}$. The characteristic band of acetone is present at $798 \mathrm{~cm}^{-1}$. The ethanol peak at $881 \mathrm{~cm}^{-1}$ makes difficult the differentiation from the peroxide peak at $878 \mathrm{~cm}^{-1}$.

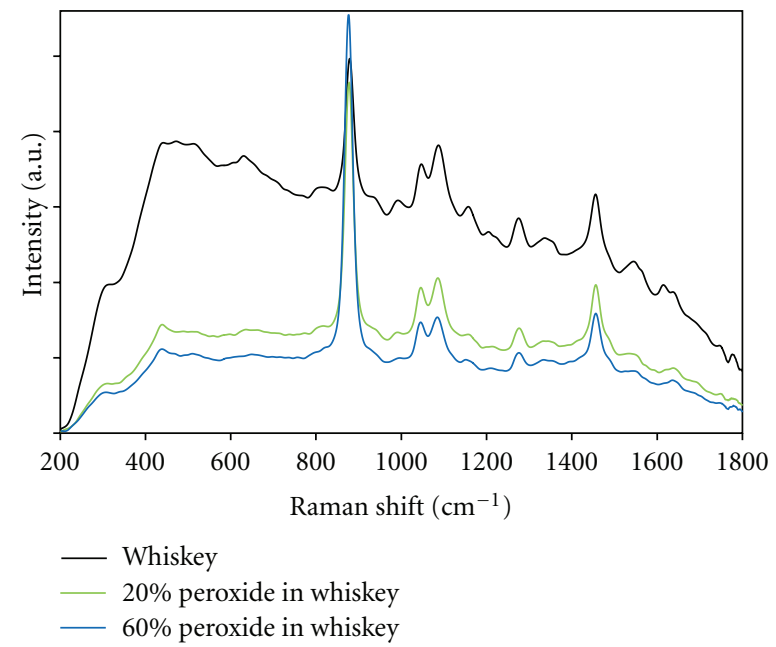

FIGURE 4: Raman spectra of a mixture of hydrogen peroxide and whiskey. Parameters were $785 \mathrm{~nm}$ laser 3 scans at $10 \mathrm{~s}$ with $200 \mathrm{~mW}$ laser power. The addition of peroxide caused no peak shifts but did increase the intensity of the band at $874 \mathrm{~cm}^{-1}$.

presents clear characteristic bands at $881 \mathrm{~cm}^{-1}, 1048 \mathrm{~cm}^{-1}$, $1086 \mathrm{~cm}^{-1}, 1280 \mathrm{~cm}^{-1}$, and $1456 \mathrm{~cm}^{-1}$ that together with known band ratios can be used to differentiate the rum from other liquids. In this case, acetone is clearly identifiable with a unique band at $789 \mathrm{~cm}^{-1}$. However the strong band at $881 \mathrm{~cm}^{-1}$ due to $\mathrm{C}-\mathrm{C}-\mathrm{O}$ stretching that all liquors present is located in the same region of the hydrogen peroxide $\mathrm{C}-\mathrm{O}$ band at $878 \mathrm{~cm}^{-1}$. This may represent a challenge for the detection of peroxide mixed with liquors.

Figure 4 shows the Raman spectra of $20 \% \mathrm{v} / \mathrm{v}$ and $60 \%$ $\mathrm{v} / \mathrm{v}$ hydrogen peroxide prepared from $50 \% \mathrm{H}_{2} \mathrm{O}_{2}$ solution in water $\left(10 \%\right.$ and $30 \%, \mathrm{H}_{2} \mathrm{O}_{2}$ resp.) in whiskey. As previously mentioned, the spectroscopic signature of peroxide is hidden 


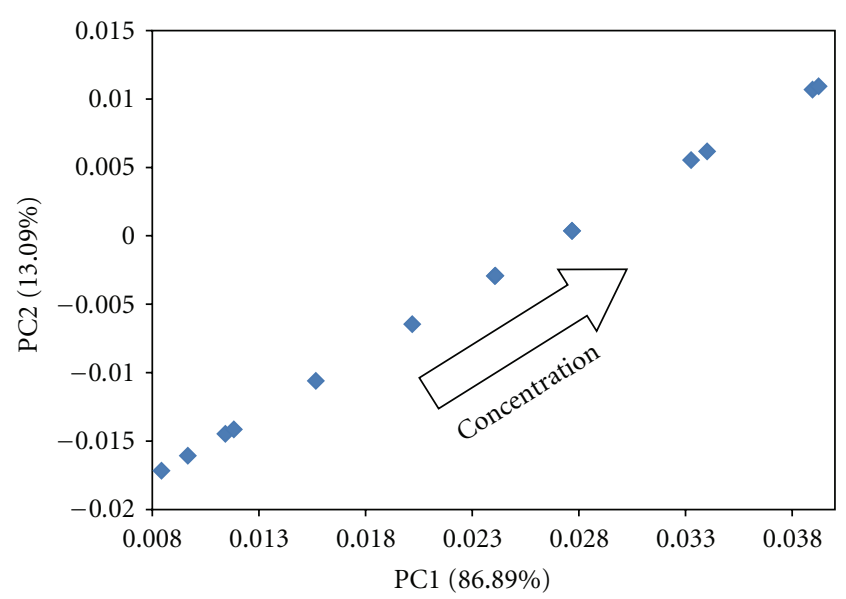

Figure 5: Scores of PC1 and PC2 (99.98\% of the spectral variance) are related to the change in concentration of hydrogen peroxide. Quantification of mixtures can be efficiently carried out using these two components only.

or masked by the ethanolic peak at $881 \mathrm{~cm}^{-1}$. Samples from $0 \%$ to $90 \%$ peroxide in whiskey were analyzed without observing changes in the location of peaks. However, the presence of peroxide has an effect on the intensity of the band $881 \mathrm{~cm}^{-1}$.

PLS was used to build a model to determine the concentration of peroxide in whiskey. The data was evaluated in the range of 200 to $1800 \mathrm{~cm}^{-1}$, after spectral intensity normalization. Figure 5 shows the scores for principal components (PC's) 1 and 2. These two components are responsible for $99.98 \%$ of the variance in the spectra. The score on each spectrum for these components confirms that there are spectral differences in the data set as a consequence of concentration of peroxide.

An evaluation of the loadings provides information on the origin of the variance captured by PC1 and PC2. Figure 6 confirms that mainly the origin of the variance captured by PC1 and PC2 is within the area of the $881 \mathrm{~cm}^{-1}$ peak. The PC2 suggests that there are contributions in the increase and the decrease of the intensity of the spectrum as well. This information is used to create a PLS regression of the spectral variance $(x)$ versus concentration of hydrogen peroxide $(y)$. This model was evaluated by a leave-one-out cross-validation. The results of the validation were evaluated by the root mean square error of calibration (RMSEC) and the root mean square error of cross-validation (RMSECV). In the first one, the values in the data set are predicted with the calibration curve obtained and in the second the values are estimated using a calibration curve that did not include this value. For the case of peroxide I whiskey, the prediction of $\%$ of peroxide versus actual concentration (Figure 7) can be achieved with a RMSEC of 2.23 and RMSECV of 2.69. The linearity of the fit is described by the $R^{2}$ value $(0.9945)$.

\subsection{Detection of Toxic Industrial Compounds in Personal Care} Products. Consumer products such as personal care and cosmetics present a challenge. Commonly these products

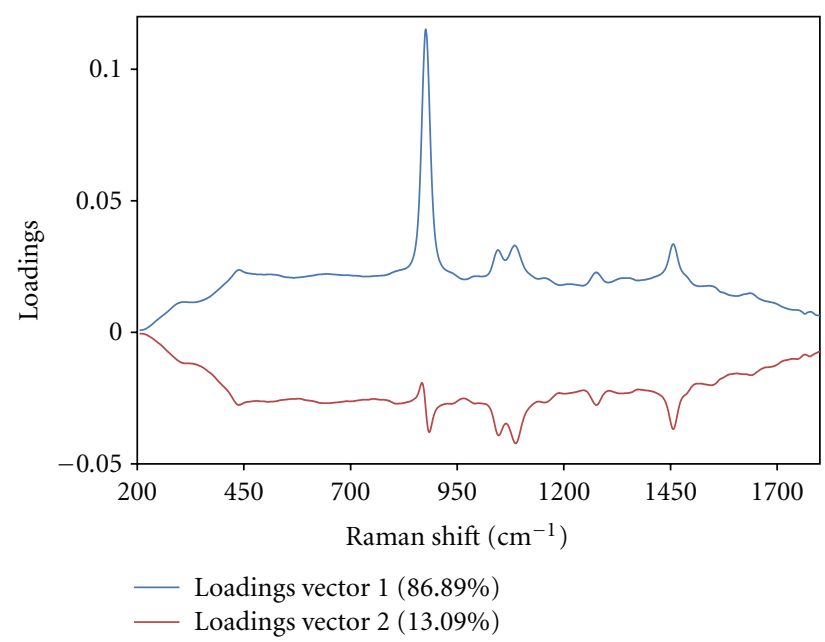

FIgure 6: Loadings vectors for PC1 and PC2. The data confirms that spectral changes in the region of the hydrogen peroxide peak $\left(878 \mathrm{~cm}^{-1}\right)$ are responsible for most of the spectral variance.

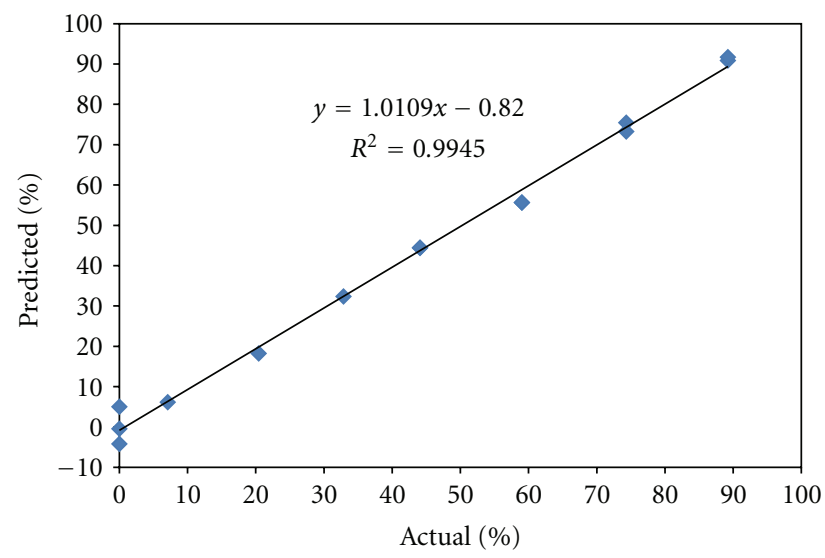

Figure 7: Prediction of the concentration of peroxide in whiskey showing a significant relation between concentration and spectral changes. This relation suggests that it is possible to discriminate between expected alcohol concentration in whisky and the presence of other material contributing to this signal.

contain multiple ingredients such as alcohols, peroxides, and others with chemical composition related to hazardous chemicals. Many personal care products are also colored and present fluorescence, obscuring the persistent Raman signatures. The spectra of a perfume is shown in Figure 8. The spectrum was obtained in its original bottle. The spectrum of $10 \%$ acetone in the perfume is included. The fluorescence and peak of the perfume dominates the signature of the liquid.

Acetone was mixed with the perfume at concentration between $10-50 \%$ (volume by volume). At higher concentrations, the spectrum of acetone becomes more evident. After a first assessment of principal components and loadings vectors, the spectral range of $600 \mathrm{~cm}^{-1}$ to $1200 \mathrm{~cm}^{-1}$ was used to estimate the concentration of acetone in the perfume. The data was first mean centered. Figure 9 presents the predicted versus actual concentration obtained with a PLS 


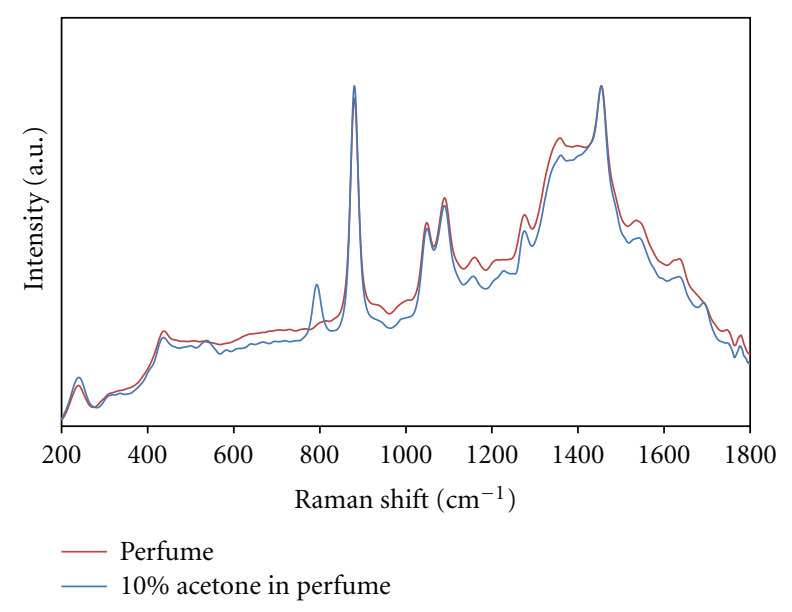

FIGURE 8: Raman spectra of a perfume and a mixture of perfume and $10 \%$ acetone. Parameters were $785 \mathrm{~nm}$ laser, $100 \mathrm{~mW}$, and $10 \mathrm{~s}$ integration time. Presence of acetone is only suggested by the small peak at $880 \mathrm{~cm}^{-1}$.

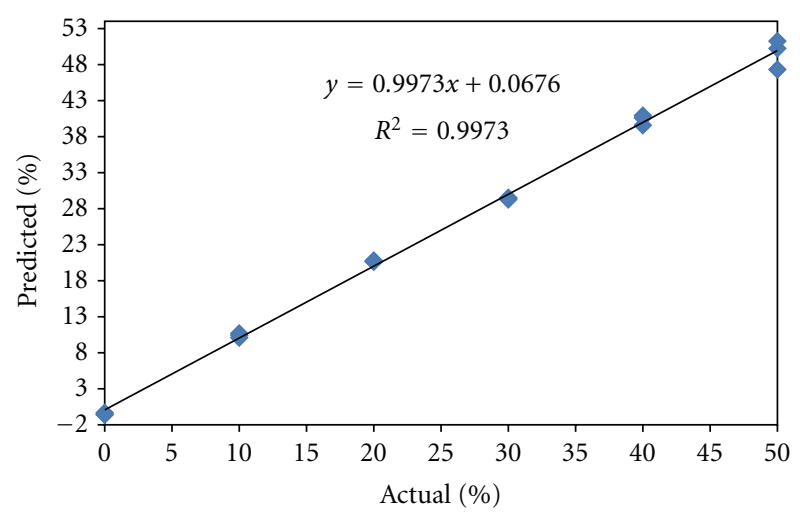

FIgure 9: PLS determination of acetone in perfume. Conditions: spectral range: 600 to $1200 \mathrm{~cm}^{-1}$, mean centering of spectra, three components (PC's) used.

based model in this spectral region. Three components were used to achieve a RMSEC of 0.887 and a RMSECV of 1.287.

3.3. Detection in Colored Liquids. The detection of a CWA simulant in heavily colored liquids was studied by FOCRS. TEP is commonly used as a simulant of Soman (GD), a nerve agent. Figure 10 presents the spectra of different flavors of Gatorade described by colors. The red presents a higher baseline associated to fluorescence. No characteristic peaks were observed for the red, blue, or orange liquids at these conditions.

TEP Raman spectrum, as shown in Figure 11, has characteristics peaks such as $733 \mathrm{~cm}^{-1}\left(\mathrm{PO}_{3}\right.$ symmetric stretch), $813 \mathrm{~cm}^{-1}\left(\mathrm{PO}_{3}\right.$ asymmetric stretch), 1032 and $1098 \mathrm{~cm}^{-1}$ (C-O stretch), $1162 \mathrm{~cm}^{-1}\left(\mathrm{CH}_{3}\right.$ rocking), and $1279 \mathrm{~cm}^{-1}(\mathrm{P}-$ O symmetric stretch) [26]. TEP was then mixed randomly with the different colors of gatorade at concentration up to $85 \% \mathrm{v} / \mathrm{v}$ of TEP. Figure 11 shows the spectrum of TEP and its mixtures with red Gatorade at concentration $6 \%$ and

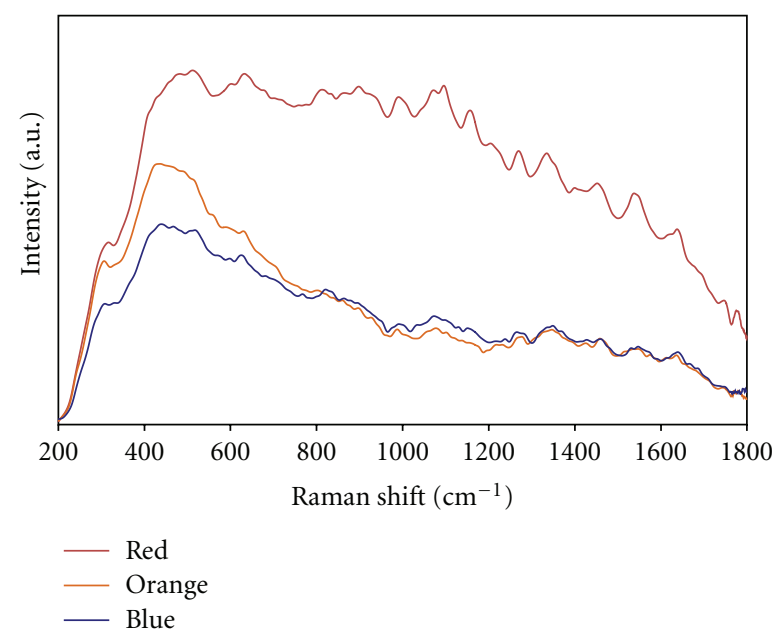

FIGURE 10: Raman spectra of red, blue, and orange Gatorade beverages. Parameters were $785 \mathrm{~nm}$ laser, $100 \mathrm{~mW}$, and $10 \mathrm{~s}$ integration time.

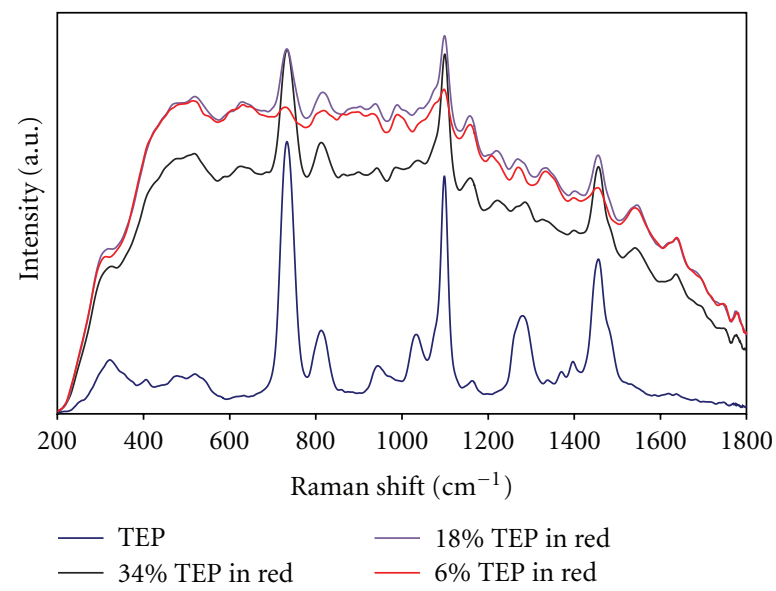

FIGURE 11: Spectrum of neat TEP and at different concentrations in red Gatorade. The spectral range of 200 to $1800 \mathrm{~cm}^{-1}$ was used for PLS-based quantification.

$34 \%$ of TEP. At concentrations higher than $50 \%$ of TEP, the signature could be easily distinguished over the spectrum of the beverage.

A discrimination exercise in which PLS was used to discriminate between samples with and without TEP was performed. For this experiment, all Gatorade samples that did not contain TEP were assigned a value of " -1 ", and those that contained TEP were labeled as "1". The samples with TEP had concentrations of the analyte in Gatorade as follows: $13 \%$ TEP in blue, $11 \%$ TEP in orange, $18 \%$ TEP in red, and $6 \%$ in red. A PLS-DA regression was performed, and the results are summarized in Figure 12. Specificity and sensitivity for each class $(y=1$ and $y=-1)$ was one (1). However, it is seen that, for samples in red Gatorade, this discrimination was not as evident as for other colors. Specifically the group of $6 \%$ TEP in red Gatorade obtained values close to zero, suggesting that this concentration of TEP is close to the detection limits of the approach. The thresholds 
TABLE 1: LOD and LOQ for TEP in colored liquids.

\begin{tabular}{lcccccc}
\hline Gatorade variety & PC's & Cumulative \% variance & $R^{2}$ of prediction curve & RMSEC & LOD & LOQ \\
\hline Red & 2 & 99.99 & 0.998 & 1.52 & 6.5 & 21.8 \\
Blue & 2 & 95.72 & 0.967 & 2.85 & 5.2 & 17.2 \\
Orange & 2 & 99.58 & 0.961 & 4.30 & 10.4 \\
\hline
\end{tabular}

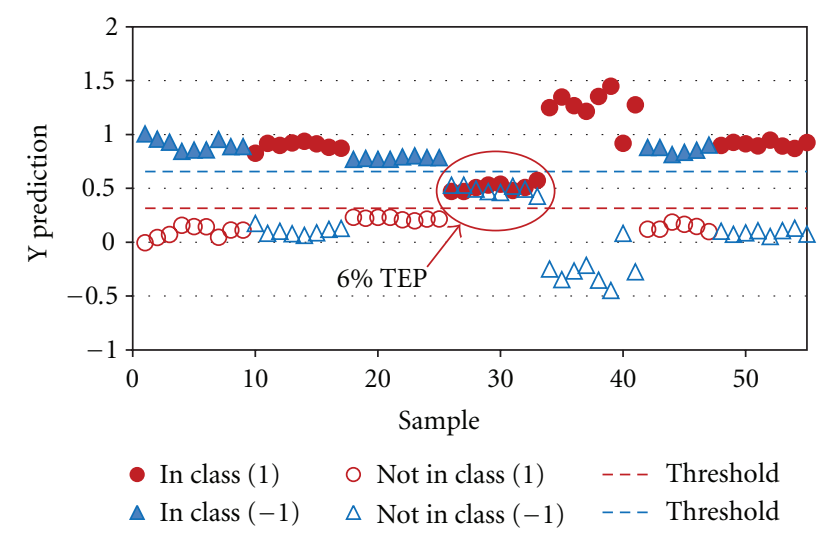

FIgUre 12: PLS-based discriminant analysis of TEP in different varieties of Gatorade. A positive value (1) implies presence of TEP, and a negative value $(-1)$ do not contain TEP. Values close to the threshold line ( $x$ axis) indicate that samples are close in spectral character making more challenging the discrimination. For $y=-1$ and 3 PC's; $R^{2} \mathrm{cal}=0.745 R^{2} \mathrm{CV}=0.728$, RMSEC $=$ 0.249 , and $\mathrm{RMSECV}=0.257$.

values for presence were 0.3156 and for not presence 0.6560 . These values represent the critical value of prediction that will separate classes. Higher values will correspond to better discrimination. In this case, samples without contamination (pure liquids) were clearly discriminated from samples with contamination at low percentage of concentration.

PLS-quantitative analysis was performed on samples containing TEP at concentration levels lower tan 50\% since the presence of TEP is more obvious over that value. Quantification was performed by individual colors. The data was mean centered and analyzed by PLS in the range of $200 \mathrm{~cm}^{-1}$ to $1800 \mathrm{~cm}^{-1}$. In order to compare the quantification, all results were compared at 2 PC's. However, improvement in the model with 3 and 4 components was observed. Data is summarized in Table 1. Results showed that quantification of TEP in these colored matrices can be achieved with good linearity and RMSECV of less than 5\%.

Finally, the limits of detection (LOD) for this analyte were calculated according to the IUPAC (International Union of Pure and Applied Chemistry) criteria of $3 \sigma$ ( 3 times standard deviation of the peak-to-peak noise related to the slope of the linear regression function). When the signal is 3 times as great as the noise, it is readily detectable but still too small for accurate measurement. A signal that is 10 times as great as the noise is defined as the lower limit of quantification (LOQ), or the smallest amount that can be measured with reasonable accuracy [27].
For this study the region from 675 to $855 \mathrm{~cm}^{-1}$ was integrated, and its relation to the concentration was evaluated by regression analysis to estimate LOD and LOQ. The objective of these graphs is to compare the limit of detection (LOD) and the limit of quantification (LOQ) between colored liquids. This region was selected because of the presence of the characteristic peaks for phosphates. This region will simulate the characteristic spectral region of real CWA and related simulants.

Table 1 presents a summary of the PLS-based detection as well as the LOD and LOQ obtained for TEP in Gatorade. The limits of detection for this analysis were at $10 \%$ or less with LOQ between 17 and 35\%. However, the low RMSEC suggests that quantification can be achieved with PLS or other algorithms at levels close to the LOD.

\section{Conclusions}

In this work, concealed liquids scenarios were studied by FOCRS. A fiber optic coupled Raman probe was used to differentiate hazardous liquids from common drinks and consumer products by acquiring spectra through the container walls. PLS algorithms were used to quantify the liquids with RMSEC of less than 5\%. These results demonstrated that Raman spectroscopy can be used as a tool to quickly determine if the content of a bottle is the intended commercial product or has been used to conceal a hazardous material. The fluorescence of a red liquid did not affect the detection of a CWA simulant in a clear glass container. The limits of detection for a hazardous liquid in a series of colored liquids were estimated in 6-10\% using standard conditions and no data manipulation. This suggests that trace level detection can be achieved with enhanced experimental setups and statistical analysis of the data.

\section{Acknowledgments}

Support from the US Department of Homeland Security under Award no. 2008-ST-061-ED0001 is acknowledged. However, the views and conclusions contained in this document are those of the authors and should not be considered a representation of the official policies, either expressed or implied, of the US Department of Homeland Security. Part of the work presented in this study was supported by the US Department of Defense University Research Initiative Multidisciplinary University Research Initiative (URI)MURI Program under the Grant no. DAAD19-02-1-0257. The authors also acknowledge contributions from Mr. Aaron LaPointe from the Night Vision and Electronic Sensors Directorate, Fort Belvoir, VA, Department of Defense, Dr. Jennifer Becker, MURI Program Manager, Army Research 
Office, DoD, and Dr. Stephen J. Lee, Chief Scientist, Science and Technology, Office of the Director, Army Research Office/Army Research Laboratory, DoD. The authors also acknowledge contributions of Omar Rivera and Gabriel Nieves and the Chemical Imaging Center at the Chemistry Department of the University of Puerto Rico-Mayagüez.

\section{References}

[1] Police: Plot to blow up aircraft foiled. http://www.cnn.com. Posted August 10, 2006.

[2] Y. Sun and K. Y. Ong, Detection Technologies for Chemical Warfare Agents and Toxic Vapors, CRC Press, Boca Raton, Fla, USA, 2005.

[3] T. C. Marrs, R. Maynard, and F. Sidell, Chemical Warfare Agents: Toxicology and Treatment, John Wiley \& Sons, London, UK, 1996.

[4] National Research Council, Final Report on Existing and Potential Standoff Explosives Detection Techniques, National Academy of Sciences, Washington, DC, USA, 2004.

[5] S. Farquharson, A. Gift, P. Maksymiuk, and F. Inscore, "Surface-enhanced raman spectra of VX and its hydrolysis products," Applied Spectroscopy, vol. 59, no. 5, pp. 654-660, 2005.

[6] K. L. McNesby, J. E. Wolfe, J. B. Morris, and R. A. Pesce-Rodriguez, "Fourier transform Raman spectroscopy of some energetic materials and propellant formulations," Journal of Raman Spectroscopy, vol. 25, no. 2, pp. 75-87, 1994.

[7] W. F. Pearman and A. W. Fountain, "Classification of chemical and biological warfare agent simulants by surface-enhanced Raman spectroscopy and multivariate statistical techniques," Applied Spectroscopy, vol. 60, no. 4, pp. 356-365, 2006.

[8] I. R. Lewis, N. W. Daniel Jr, N. C. Chaffin, P. R. Griffiths, and M. W. Tungol, "Raman spectroscopic studies of explosive materials: towards a fieldable explosives detector," Spectrochimica Acta Part A, vol. 51, no. 12, pp. 1985-2000, 1995.

[9] M. Donahue, H. Huang, C. Brouillette, W. Smith, and S. Farquharson, "Detecting explosives by portable Raman analyzers: a comparison of 785, 976, 1064, and $1550 \mathrm{~nm}$ (RetinaSafe) laser excitation," Spectroscopy, pp. 24-28, 2011.

[10] S. Christesen, B. Maciver, L. Procell, D. Sorrick, M. Carrabba, and J. Bello, "Nonintrusive analysis of chemical agent identification sets using a portable fiber-optic Raman spectrometer," Applied Spectroscopy, vol. 53, no. 7, pp. 850-855, 1999.

[11] S. D. Harvey, M. E. Vucelick, R. N. Lee, and B. W. Wright, "Blind field test evaluation of Raman spectroscopy as a forensic tool," Forensic Science International, vol. 125, no. 1, pp. 12-21, 2002.

[12] C. Eliasson, N. A. Macleod, and P. Matousek, "Noninvasive detection of concealed liquid explosives using Raman spectroscopy," Analytical Chemistry, vol. 79, no. 21, pp. 8185-8189, 2007.

[13] C. Eliasson, N. A. Macleod, and P. Matousek, "Non-invasive detection of powders concealed within diffusely scattering plastic containers," Vibrational Spectroscopy, vol. 48, no. 1, pp. 8-11, 2008.

[14] M. Alvarez-Rivera, Fiber optic coupled Raman spectroscopy of liquid explosives mixtures, M.S. Dissertation, University of Puerto Rico, Mayaguez, Puerto Rico, 2002.

[15] C. Frausto-Reyesa, C. Medina-Gutierrez, R. Sato-Berrub, and L. R. Sahaguna, "Qualitative study of ethanol content in tequilas by Raman spectroscopy and principal component analysis," Spectrochimica Acta Part A, vol. 61, no. 11-12, pp. 2657-2662, 2005.
[16] J. D. Driskell, Y. Zhu, C. D. Kirkwood, Y. Zhao, R. A. Dluhy, and R. A. Tripp, "Rapid and sensitive detection of rotavirus molecular signatures using surface enhanced raman spectroscopy," PLoS ONE, vol. 5, no. 4, Article ID 10.1371/journal.pone.0010222, 2010.

[17] V. Sikirzhytski, K. Virkler, and I. K. Lednev, "Discriminant analysis of Raman spectra for body fluid identification for forensic purposes," Sensors, vol. 10, no. 4, pp. 2869-2884, 2010.

[18] W. O. Georgeand and D. Steele, Computing Applications in Molecular Spectroscopy, Royal Society of Chemistry, Cambridge, UK, 1995.

[19] O. Svensson, M. Josefson, and F. W. Langkilde, "Reaction monitoring using Raman spectroscopy and chemometrics," Chemometrics and Intelligent Laboratory Systems, vol. 49, no. 1, pp. 49-66, 1999.

[20] E. K. Plyler, "Infrared spectra of methanol, ethanol, and npropanol," Journal of Research of the National Bureau of Standards, vol. 48, no. 4, pp. 281-286, 1952.

[21] N. A. Milas and A. Golubović, "Studies in organic peroxides. XXVI. Organic peroxides derived from acetone and hydrogen peroxide," Journal of the American Chemical Society, vol. 81, no. 24, pp. 6461-6462, 1959.

[22] L. Jensen, P. M. Mortensen, R. Trane, P. Harris, and R. W. Berg, "Reaction kinetics of acetone peroxide formation and structure investigations using Raman spectroscopy and X-ray diffraction," Applied Spectroscopy, vol. 63, no. 1, pp. 92-97, 2009.

[23] G. Dellepiane and J. Overend, "Vibrational spectra and assignment of acetone, $\alpha \alpha \alpha$ acetone- $\mathrm{d} 3$ and acetone- $\mathrm{d} 6$," Spectrochimica Acta, vol. 22, no. 4, pp. 593-614, 1966.

[24] J. Oxley, J. Smith, J. Brady et al., "Raman and infrared fingerprint spectroscopy of peroxide-based explosives," Applied Spectroscopy, vol. 62, no. 8, pp. 906-915, 2008.

[25] L. Pacheco-Londoño, O. M. Primera, M. Ramírez, O. Ruiz, and S. Hernandez-Rivera, "Review of the various analytical techniques and algorithms for detection and quantification of TATP," in Proceedings of the international society for optics and photonics (SPIE '05), E. M. Carapezza, Ed., vol. 5778, pp. 317326, Sensors, and C3I Technologies for Homeland Security and Homeland Defense IV, Bellingham, Wash, USA, month year.

[26] K. Taga, N. Hirabayashi, T. Yoshida, and H. Okabayashi, "Raman spectra and rotational isomerism of dimethylphosphorochloridate and trimethyl phosphate," Journal of Molecular Structure, vol. 212, pp. 157-168, 1989.

[27] V. Thomsen, D. Schatzlein, and D. Mercuro, "Limits of detection in spectroscopy," Spectroscopy, vol. 18, no. 12, pp. 112114, 2003. 


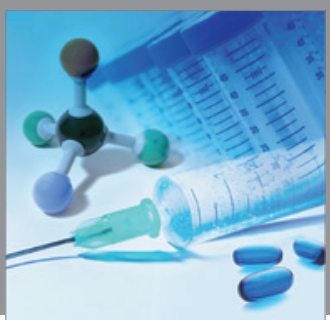

International Journal of

Medicinal Chemistry

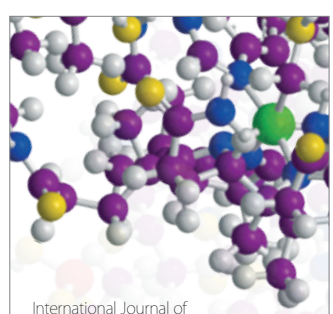

Carbohydrate Chemistry

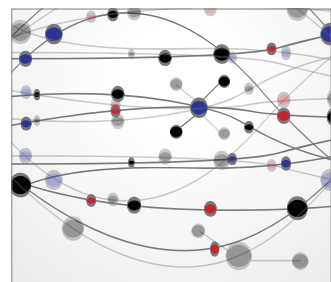

The Scientific World Journal
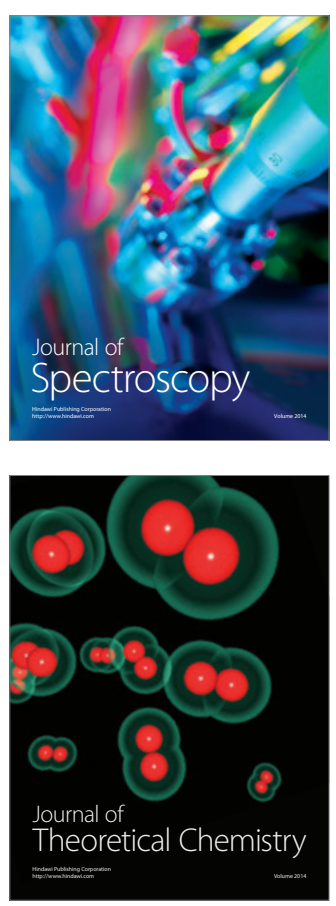
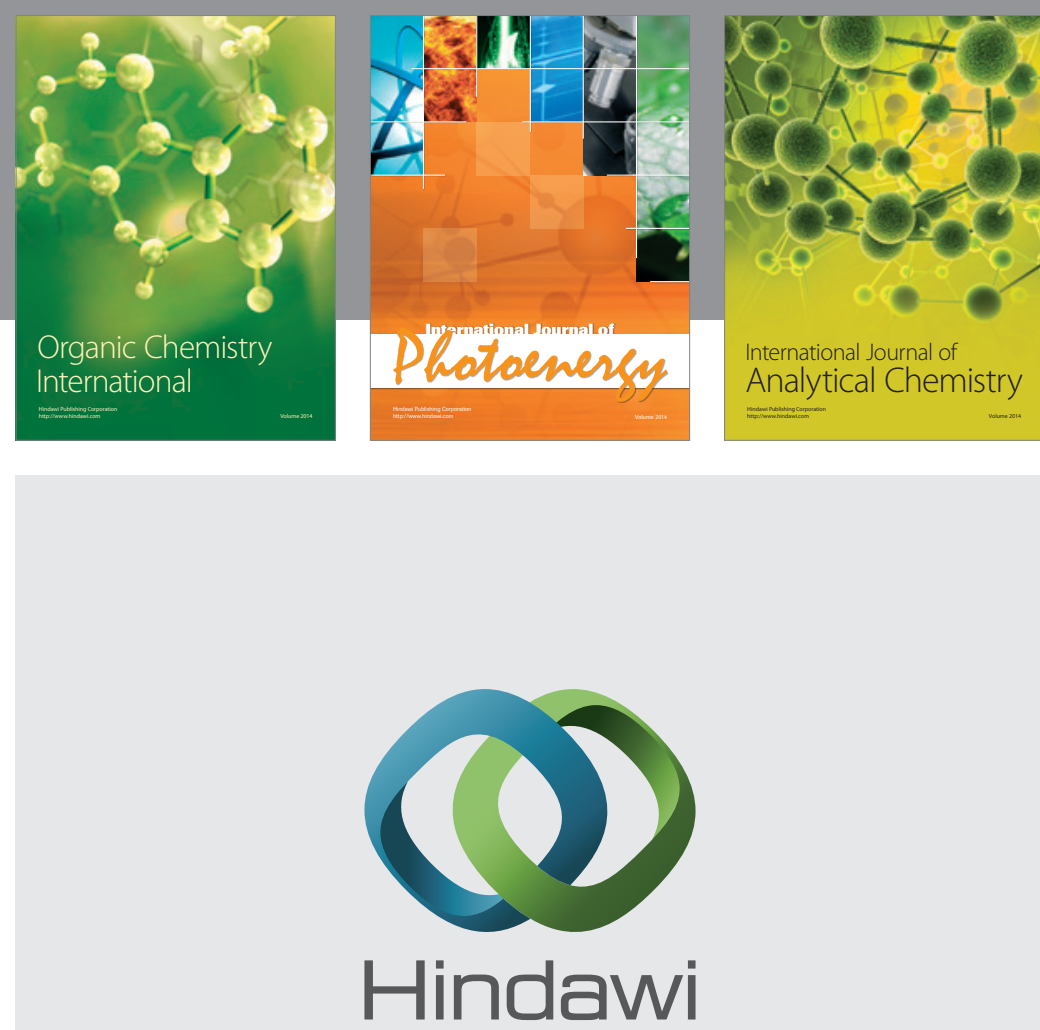

Submit your manuscripts at

http://www.hindawi.com
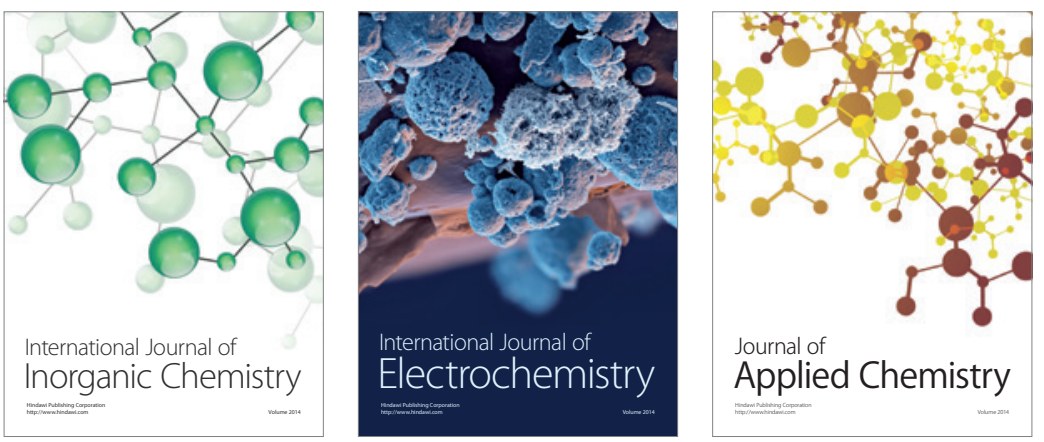

Journal of

Applied Chemistry
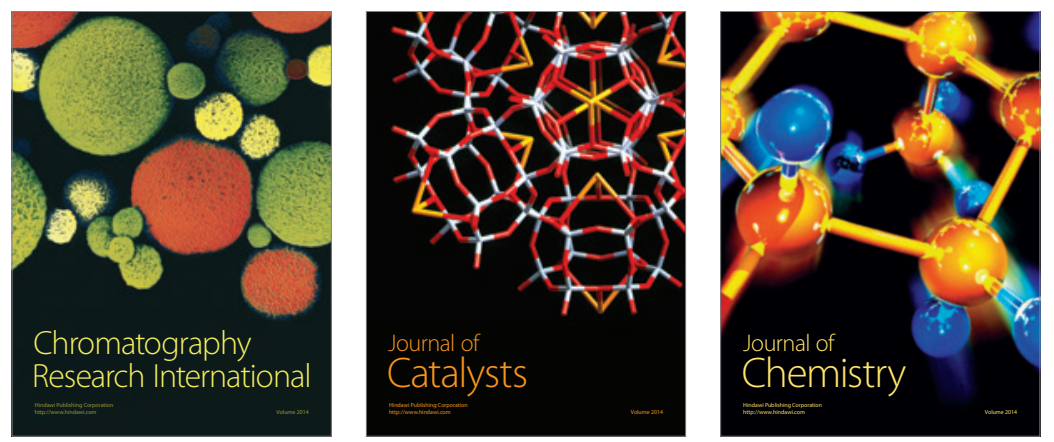
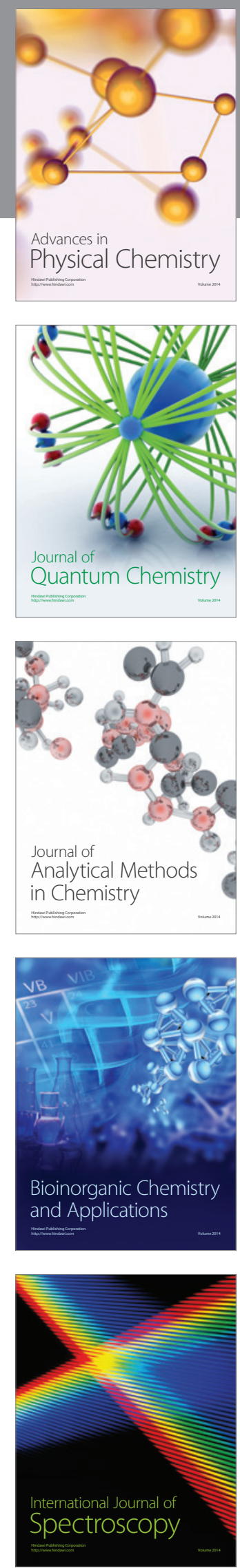